\title{
Inspiration and Pre-Conception of an Intelligent and Dynamic System Supporting Learner Ontologies
}

\author{
Mehdi TMIMI $^{\# 1}$, Mohamed BENSLIMANE ${ }^{* 2}$, Mohammed BERRADA ${ }^{\# 3}$, Kamar OUAZZANI ${ }^{\# 4}$ \\ ${ }^{\#}$ Laboratory of computing and interdisciplinary physics, ENS, USMBA, Fez, Morocco \\ ${ }^{1}$ mehdi.tmimi@usmba.ac.ma \\ ${ }^{3}$ mohammed.berrada@gmail.com \\ ${ }^{4}$ kamar_ouazzani@yahoo.fr \\ * Transmission and Treatment of Information Laboratory, EST, USMBA, Fez, Morocco \\ ${ }^{2}$ benslimane_mohamed@live.fr
}

\begin{abstract}
Learner ontologies are a growing and top actuality theme in the work of adaptive hypermedia.

The context of this article is to focus on the major Problematic of learner ontologies, which is that they are not dynamic and evolutive, in the sense that ontologies are like elements with values that differ from a learner To the other however human nature is far from being as simple as that.

To respond to this problematic, we are being inspired from human nature (learner) itself. And we offer a scalable, flexible system that will imitate human complexity in order to present and describe the learning profile in every detail.
\end{abstract}

Keyword-Ontology, Learner profile, Adaptive hypermedia, Neuron, brain, E-learning, Domain model, Adaptation model.

\section{INTRODUCTION}

E-learning is the use of new multimedia technologies and the Internet to improve the quality of learning by facilitating access to resources and services, as well as exchanges and collaboration from distance (European Commission, 2001) "as stated in [1]", witch allow the classrooms and platforms to be independent [2] and enables distributed e-learners to access various learning resources much more convenient and flexible [3].

Several approaches and systems (LMS, MOOC...) have taken the step towards E-learning and have dominated Internet education over the last two decades. Among these approaches we quote our previous article 'Proposal of an Approach of Online Course Design and Implementation: A Case Study of an Algorithmic Course' [4] where we proposed an online course approach based on a solid set of specifications for our course (algorithmic course), and ending with its implementation on the LMS (Learning Management System) Moodle.

However, these 'traditional' systems do not respect the progress made in Internet technologies and online social interactions [5], and apply the philosophy of "one-size-fits all" which means the same document is presented to all learners, so they do not have the same needs nor the same knowledge, nor the same learning styles [6].

As a result, a new generation of advanced learning systems has emerged by giving the learner an active role to learn and build his knowledge. These systems are highly interactive and integrate a more user-centric vision [7].

Adaptive hypermedia systems offer solutions to these problems and can be described as adaptive e-learning and more precisely as Brusilovsky has defined it: "An Adaptive Hypermedia System is a hypertext or hypermedia system that reflects certain characteristics of the user in a user model, and applies that model to adapt certain visible aspects of the system to that user." (P. Brusilovsky) [8].

\section{INSPIRATION \& PROJECTION}

To guide ourselves in our reasoning, we have thought deeply about human nature, which is very complex in itself.

The majority of learner information resides in his memories, experiences, abilities, and knowledge ... all this is managed mainly by the human brain.

The human brain is very organized, it is composed of several parts that each have specific roles while they are being complementary to one another, and if we project the brain on the learner ontology, we will find that this organization in parts of the Brain resemble to the different facets of the learner ontology cited in a previous work (M. Tmimi) [12]. 
This complementarity (communication) is done by neurons. In fact the total number of neurons was once estimated at 85 billion (Williams and Herrup, 1988) [13], but recent studies (Andersen et al., 1992) [14] estimate a total of 105 billion neurons in the human cerebellum alone [15].

Let's see what a neuron looks like (0).

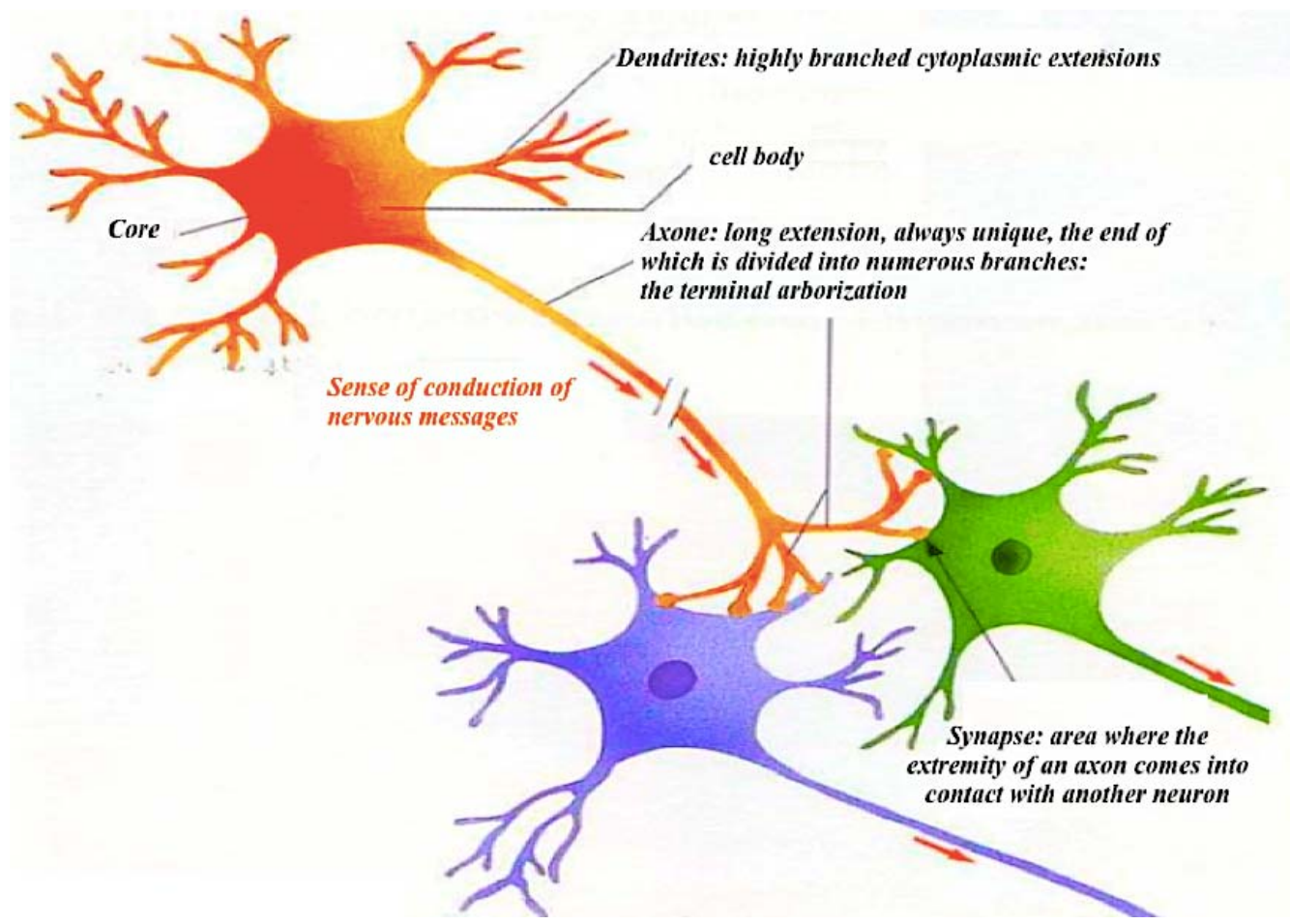

Fig. 1. The different elements of a neuron and how the communication is done [16].

It should be noted that each neuron has a cell body, dendrites and an axon, and that the communication between two neurons is done by chemical and electrical processes, the electrical nervous messages that arrive at the end of the nerves are found in a neuron, and trigger a secretion of chemical molecules that will deposit on the next neuron, where they will be translated again into an electrical message in the other neuron [16].

Reformulating this complexity while making a projection on our field of research:

- Each neuron represents information: this will be translated by an element of our ontology;

- Each neuron contains dendrites and an axon which serve as a way of communication: this will be translated by the fact that each element of our ontology (neuron) will have relations by means of inputs (dendrites) and outputs (axons).

It should also be mentioned that neurons have the ability to learn, depending on the type of learning the connections between the neurons involved are either:

- More and stronger (as shown in 0);

- Fewer and weaker.

So, the strength of connections between neurons can change quickly and last a long time [17]. 


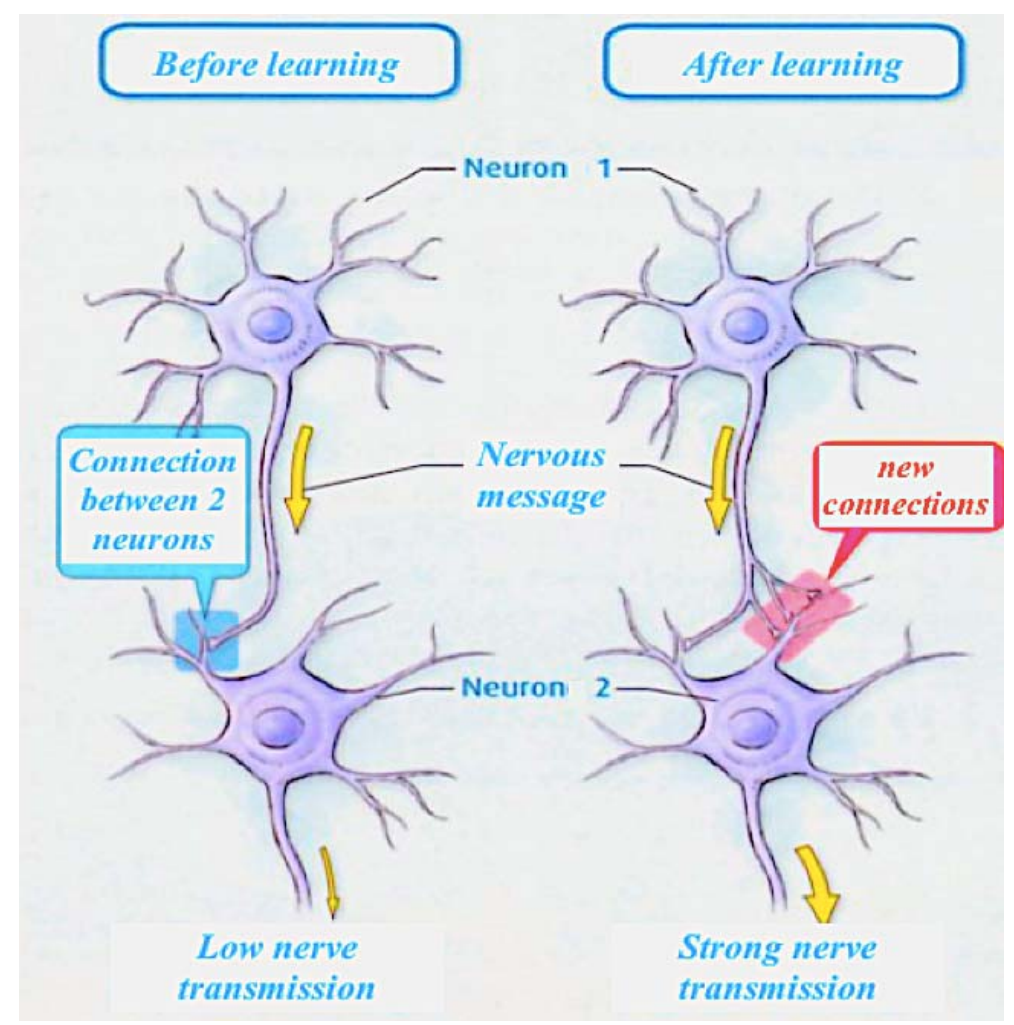

Fig. 2. Neurons before and after learning [18]

From the mechanism explained above and the previous projection, we have thought of a system that will manage the relations (connections) between the elements of our ontology based mainly on the neuron communication system.

\section{III.OUR PROPOSITION}

Our system will be in the form of a network (unique for each learner) that keeps growing according to the behaviour of the learner.

We have modelled this system with the class diagram -UML2- and to detail it we will first present the different components of our system (Class) and then its relations and finally the constraints and the rules.

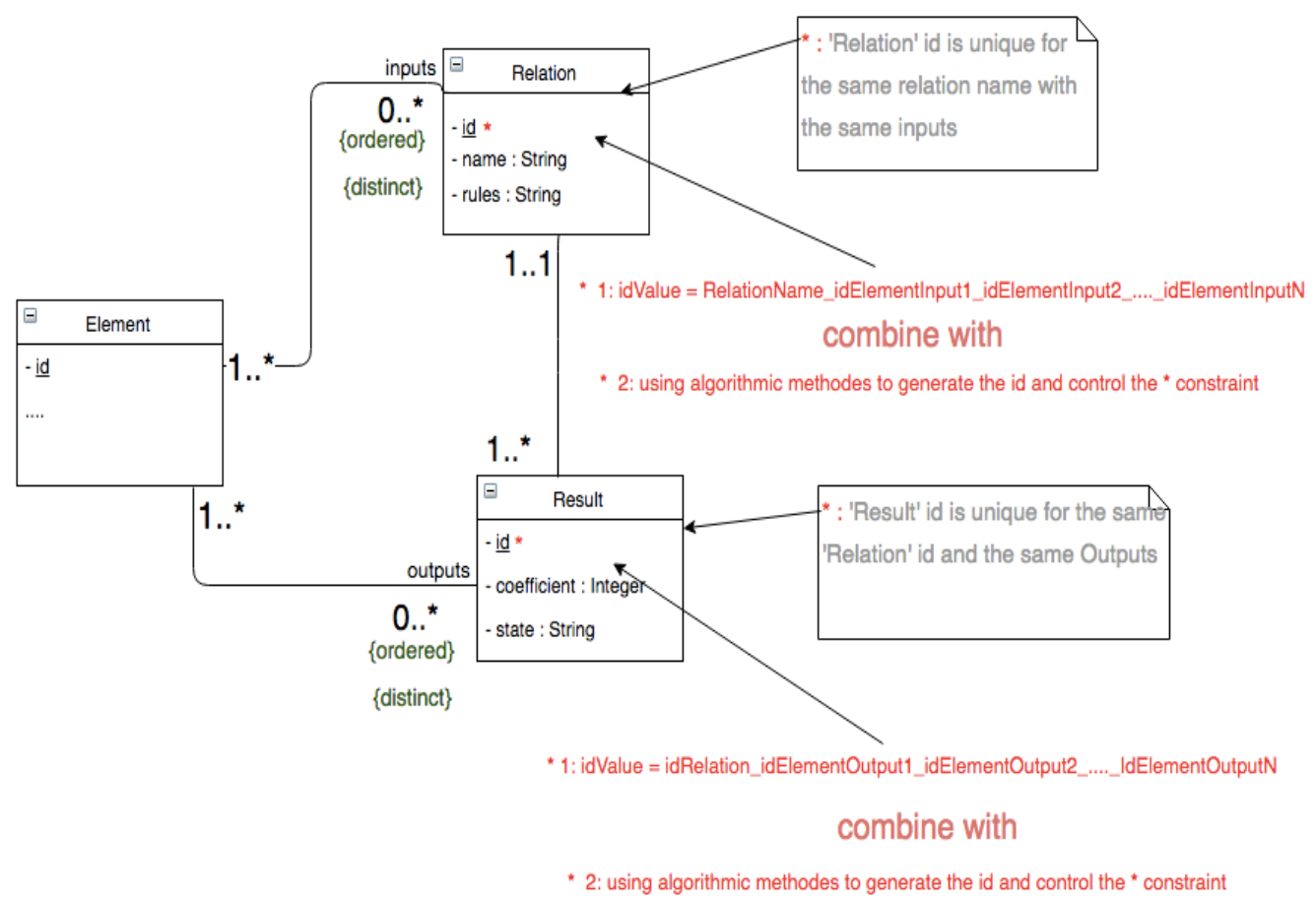

Fig. 3. Class diagram of our proposed system 


\section{A. Components (Class)/Relations}

Our System is composed of three classes, which are:

-'Element' Class: represents information of our ontology and is characterized by a unique [id] that will reference this information in the learner ontology. Each instance (object) of the 'Element' class can simultaneously play [inputs] roles for the 'Relation' class and [outputs] roles for the 'Result' class.

-'Relation' class: characterized by the attribute [id], the [name] attribute and the [rules] attribute that defines the rules and operations to be performed for all 'Result' entered \{Update, Delete, no Action\}. An instance of the 'Relation' class can have one or more 'Result', and an instance of 'Result' concerns one and only one 'Relation'.

-'Result' class: characterized by 'coefficient' which is an integer value which varies from 1 to $\infty$ depending on the number of times that the learner's behaviour has to approve this result: 'Result'. And a 'state' that is a string describing the state of this relationship \{current, historic ...\}.

\section{B. Constraints and Rules}

We will describe in this part the constraints and the rules of our system:

1) Relation: is defined by [name] and a set of [Inputs], which are (inputs: 'Elements') distinct and ordered. However, it is absolutely forbidden to have two 'Relation' with the same [name] with the same [Inputs].

In ' 0 ' we have a 'Relation: $\{1\}$ ' which has [name]: \{define $\}$ and [inputs]: $\{\mathrm{e} 1, \mathrm{e} 2\}$, and by involving the constraint cited above the 'Relation: $\{4\}$ ' will not be created and it will be considered as 'Relation: $\{1\}$ '.

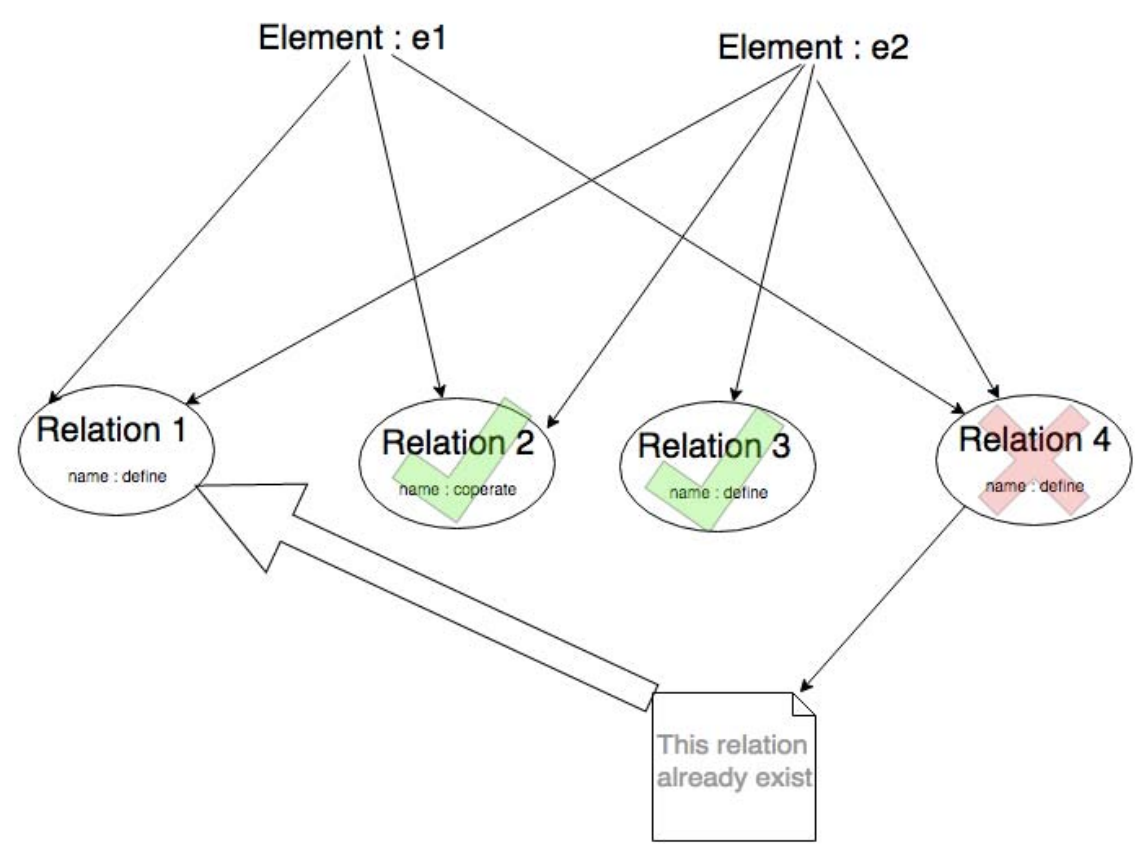

Fig. 4. Diagram expressing the constraint of the id Relation

2) Result: concerning a 'Relation' is unique for the same [outputs], which means it's forbidden to have two 'Result' with the same [outputs] and the same 'Relation'.

In ' 0 ' we have a demonstrative example of this constraint. When the learner approves an already existing 'Result', this translates to an increment of the coefficient, instead of creating a new 'Result'. 


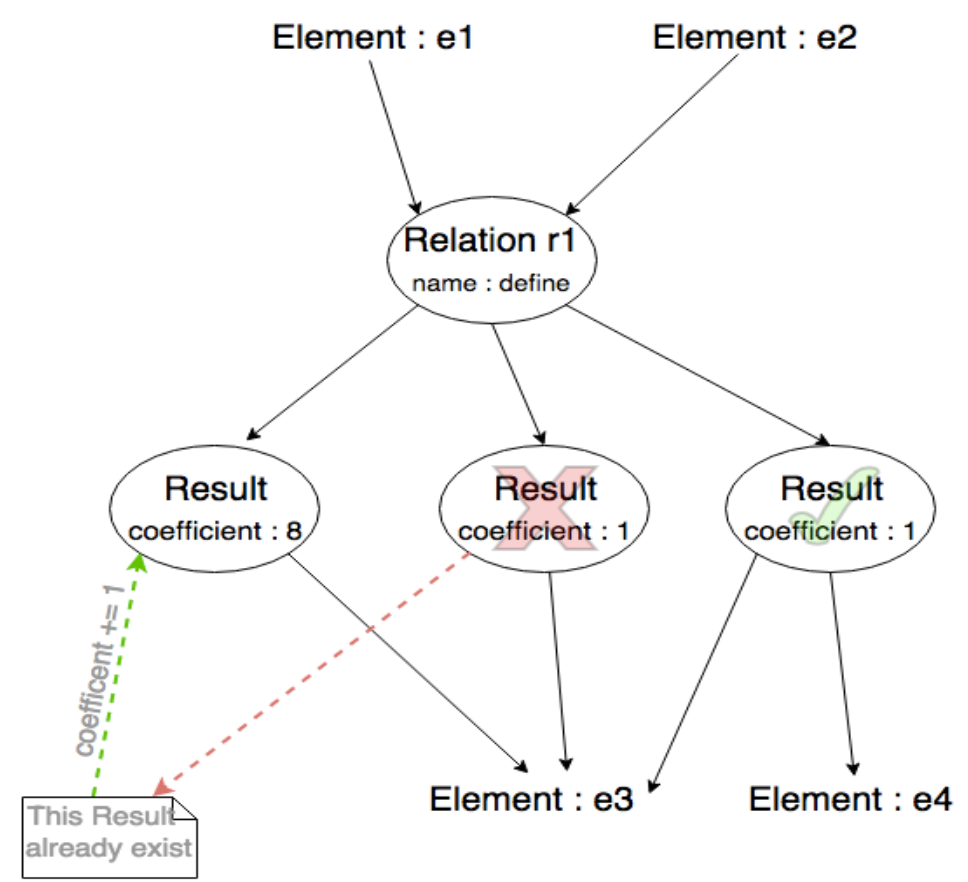

Fig. 5. Diagram expressing the constraint of the id Result

The system of coefficients will allow us to identify the 'Result' that are persistent, while keeping track of the new 'Result' whose coefficients are constantly increasing. In short if we have a 'Relation' X at a given time T1, the system will take into consideration the 'Result' with the highest coefficient.

\section{Implementing the Constraints}

The major difficulty in expressing these constraints is summarized in how to ensure the uniqueness of the 'Relation' and the 'Result' (marked by '*' in the class diagram of our proposed system -Fig 3-).

In order to ensure their uniqueness we thought of using the logical model of data where we could speak of primary key $\rightarrow$ uniqueness.

So we just have to find a way to generate the id (primary key), this generation will be done by algorithms in the following way.

1) Uniqueness of 'Relation': Id Relation will be a character string concatenation of the name of the relation with a sorted sequence of the ids of the incoming elements [Inputs].

Id 'Relation' $\leftarrow$ RelationName+_IdElement1+...+_ IdElementN

Where:

RelationName: name of the 'Relation' (define, cooperate with...);

+: Character string concatenation;

IdElement: id of the incoming elements [Inputs].

NB: The order of the elements [inputs] is very important; otherwise we will have different ids (Relation) with the same [Inputs]. This is the reason why we added \{ordered\} in the class diagram (0).

Thus, 'Element' cannot simultaneously play the same role [Input] for the same 'Relation' (no redundancy) \{distinct\} (0).

At the moment when the learner approves or triggers a 'Relation', our system will first generate the id dedicated to this 'Relation'. Then, from this generated id, the system will check if we already have a relationship with the same id, and following this verification our system will decide either to create a new one or to use the existing relationship.

2) Uniqueness of 'Result': Id Result will be a character string concatenation of the id Relation with a sorted sequence of the ids of the output elements [outputs].

Id 'Result': $\leftarrow$ IdRelation+_IdElement1+..+_ IdElementN

Where:

IdRelation: id 'Relation' generated previously.

+: Character string concatenation

IdElement: id of the output elements [outputs]. 
NB: the order of the elements [outputs] is very important; otherwise we will have different ids (Result) with the same [Outputs]. This is the reason why we added \{ordered\} in the class diagram (0).

Therefore, 'Element' cannot simultaneously play the same role [Output] for a 'Relation' (no redundancy) \{distinct\} (0).

When the learner approves or triggers a 'Result', our system will first generate the id dedicated to this 'Result'. Then from this generated id, the system will check if we already have a 'Result' with the same id, and following this check our system will either decide to create a new 'Result' and initialize the coefficient to 1 or use The 'Result' already existing without forgetting to increment the coefficient.

\section{IV.Conclusion And Perspectives}

Our system will allow existing ontologies to become more dynamic and lively, which will facilitate the learner's evolution and represent it more accurately and precisely.

Thus, since our system has a great flexibility, we are thinking of expanding its scope to integrate domain ontology and adaptation models, in order to have a unique and flexible system supporting the adaptive hypermedia that will be the Fruit of our research.

\section{REFERENCES}

[1] Commission des communautés européennes, Communication de la Commission au Conseil et au Parlement européen - Plan d'action eLearning - Penser l'éducation de demain. Bruxelles, COM (2001)172 final, le 28/03/2001.

[2] Mahnane, L., et al : A Model of Adaptive e-learning Hypermedia System based on Thinking and Learning Styles. Int. J. Multimedia Ubiquitous Eng. 8(3), 339-350 (2013).

[3] M. Yaqub , A. M. Raid, Haitham A. EL-Ghareeb : Adaptive E-Learning System Based On Learning Interactivity , International journal of Computer Science \& Network Solutions, April.2014-Volume 2. No4

[4] M. Benslimane, K. Ouazzani, M. Tmimi, M. Berrada, "Proposal of an Approach of Online Course Design and Implementation : A Case Study of an Algorithmic Course" , International Journal of Computer Technology \& Applications, Vol 7(4), 530-536, JulyAugust 2016.

[5] D. Dagger, A. O'Connor, S. Lawless, E. Walsh, VP. Wade, "Service-oriented e-learning platforms: From monolithic systems to flexible services", IEEE Internet Computing, Volume: 11, Issue: 3, May-June 2007.

[6] J. El Bouhdidi, M. Ghailani, A.Fennan "Towards an Optimized Design of Individualized Learning Paths: an Approach Based on Ontology and Multi-agents System”, International Journal Of Computer Science Issues, Vol.9, Issue 6, No 3, November 2012.

[7] A. Behaz, M. Djoudi, "Approche de Modélisation d'un Apprenant à base d'Ontologie pour un Hypermédia adaptatif Pédagogique", In: CIIA, 2009.

[8] P. Brusilovsky, "Methods and Techniques of Adaptive Hypermedia, In Adaptive Hypertext and Hypermedia", Kluwer Academic Publishers, Netherlands, pp. 1- 43, 1998.

[9] V. Carchiolo, Al. Longheu, M. Malgeri, G. Mangioni, "An Architecture to Support Adaptive E-Learning", International Journal of Computer Science and Network Security, Vol.7, No.1, 2007.

[10] D. Paneva, "Ontology-based Student Modeling", In the Proceedings of the Open Workshop "Ubiquitous Learning Challenges: Design, Experiments and Context Aware Ubiquitous Learning", Turin, Italy, pp. 17-21, 20 - 21 September 2006.

[11] O. Zine, A. Derouich, A. Talbi, "Ontologie de l'apprenant basée sur le web sémantique pour la description des préférences et styles d'apprentissage". 1 ère Edition du Workshop International sur les Approches Pédagogiques \& E-Learning. Appel'2015.

[12] M. Tmimi, M. Benslimane, M. Berrada, K. Ouazzani, "Elaboration d'une ontologie apprenant pour les hypermédias adaptatifs", Conférence Internationale sur les Approches Pédagogiques \& E-Learning, Appel'2016.

[13] Williams RW, Herrup K (1988) The control of neuron number. Annu Rev Neurosci 11:423-453.

[14] Andersen BB, Korbo L, Pakkenberg B (1992) A quantitative study of the human cerebellum with unbiased stereological techniques. J Comp Neurol 326:549-560

[15] Suzana Herculano-Houzel ,Roberto Lent : Isotropic Fractionator: A Simple, Rapid Method for the Quantification of Total Cell and Neuron Numbers in the Brain, The Journal of Neuroscience, March 9, 2005 - 25(10):2518 -2521

[16] La 3D: Cerveau, online in: "http://tpela3d.e-monsite.com/pages/i-la-vision-de-l-homme/cerveau.html".

[17] A. Guérin, J. Gaubil , A. Guesdon, Site "Amnésies et mémoire", l'établissement Jean-Paul II à Saint-Grégoire, janvier 2016.

[18] Editeur Belin, Svt, 1ere s, Manuel de l'élève (édition 2011) DUCO, ANDRE, 2011. 